\title{
Longwave IR focal-plane binary optics
}

\author{
Z. Sikorski, H. Polakowski \\ Institute of Optoelectronics, Military University of Technology, \\ 2 Kaliskiego Str., 00-908 Warsaw, e-mail: zsikorsk@wat.waw.pl
}

\begin{abstract}
In this paper we discuss a choice between the diffractive and refractive binary micro-lenses for the longwave IR FPA. To investigate diffraction on the micro-lenses we have used a numerical code based-on the rigorous electromagnetic theory, because neither geometrical optics nor thin element approach of scalar diffraction theory can correctly describe waveguide, shadowing and interference effects inside the micro-lens surface relief. These effects strongly influence the micro-lens diffraction efficiency when the width of the surface-relief features is close to the wavelength.
\end{abstract}

\section{Introduction}

Parameters of focal-plane arrays (FPA) longwave ( $8-12 \mu \mathrm{m}$ ) infrared (LWIR) uncooled detectors can be increased by replacing the detector in conventional array with a micro-lens of the same area and detector of the reduced area. This results in reduction of the noisy thermal generation rate. Binary optics is a rapidly developing theory and technology of making surface-relief micro-optics using high-performance equipment of the electronics industry. In the most popular version of binary optics technology, calculated surface-relief pattern is coded in lithography masks and subsequently transferred into the substrate relief using photolithography and dry etching. Staircase $\left(2^{m}\right.$ levels are made using $m$ masks) approximations of the micro-lens surface can be obtained both for the diffractive and refractive micro-lenses.

\section{Electromagnetic analysis of binary diffractive micro-lenses}

Rigorous electromagnetic theory and experiment predict resonant dependence of the micro-lens diffraction efficiency on the wavelength, incidence angle and polarization of light, as well as on the grooves local period and depth [1,2].

Fig. 1 shows the magnitude of the electric field inside the $\mathrm{CdZnTe}$ surface relief groove calculated using rigorous eigenmode method [4] fused with the scattering matrix approach [5, 6] for normal incidence and TE polarization of plane wave illumination. According to commonly used scalar diffraction theory, the field distribution should be homogenious inside the grooves. Strong spatial variations of the electric field presented in Fig. 1 confirm necessity of using electromagnetic theory for modeling of fast micro-lenses. The scalar theory predictions are clearly incorrect in this case. Moreover, the field distribution is dependent on the polarization of the incident field. The magnetic field distribution in the same diffractive structure as in the case presented in Fig. 1 is shown in Fig. 2 for the TM polarization of the incident wave.

The first-order diffraction efficiency of outer parts of high-numerical-aperture binary diffractive micro-lens rapidly decreases due to the shadowing and waveguiding effects, when the lens-groove width $d$ becomes smaller than $d_{\min }$, a value close to the wavelength in air and a few times higher than wavelength in the lens substrate [2]. This sets an upper limit on the 
numerical aperture of efficient binary diffractive micro-lens [3]. For the CdZnTe binary micro-lens presented in Fig. 3 this limit of efficiency occurs for numerical aperture equal to 0.9 (see Fig. 4).

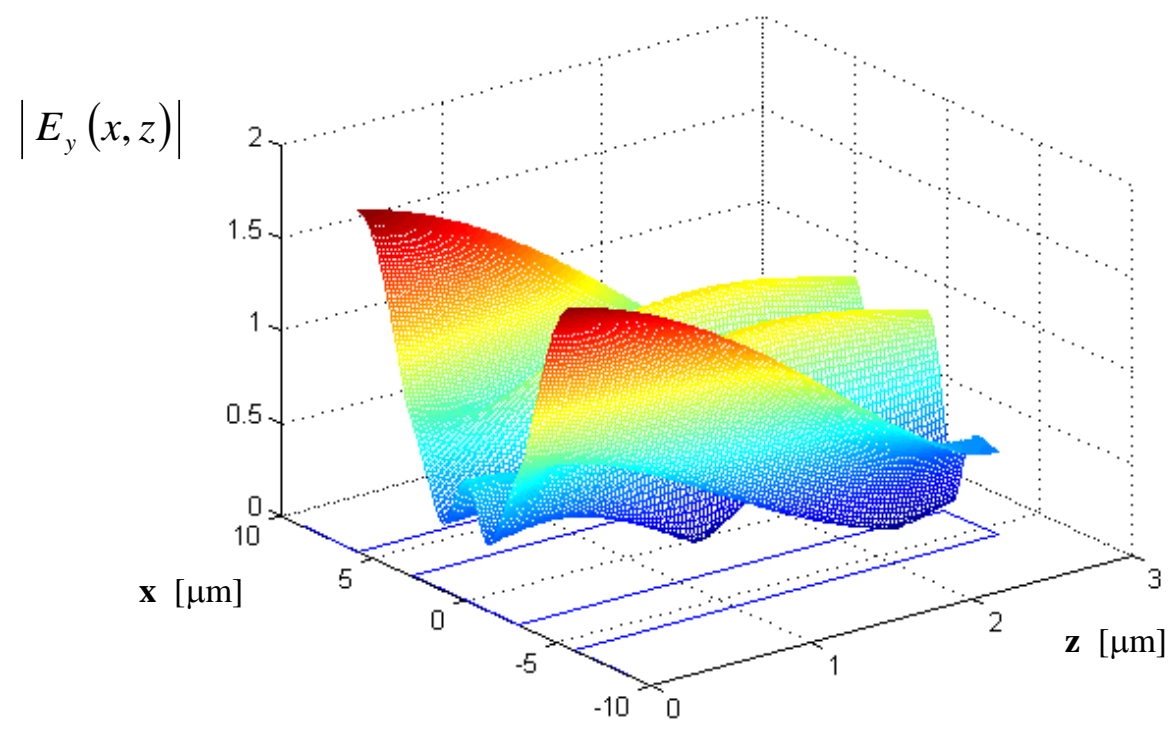

Fig. 1 Electric field magnitude inside the micro-lens groove.

The groove profile is shown in the horizontal plane. Polarization TE

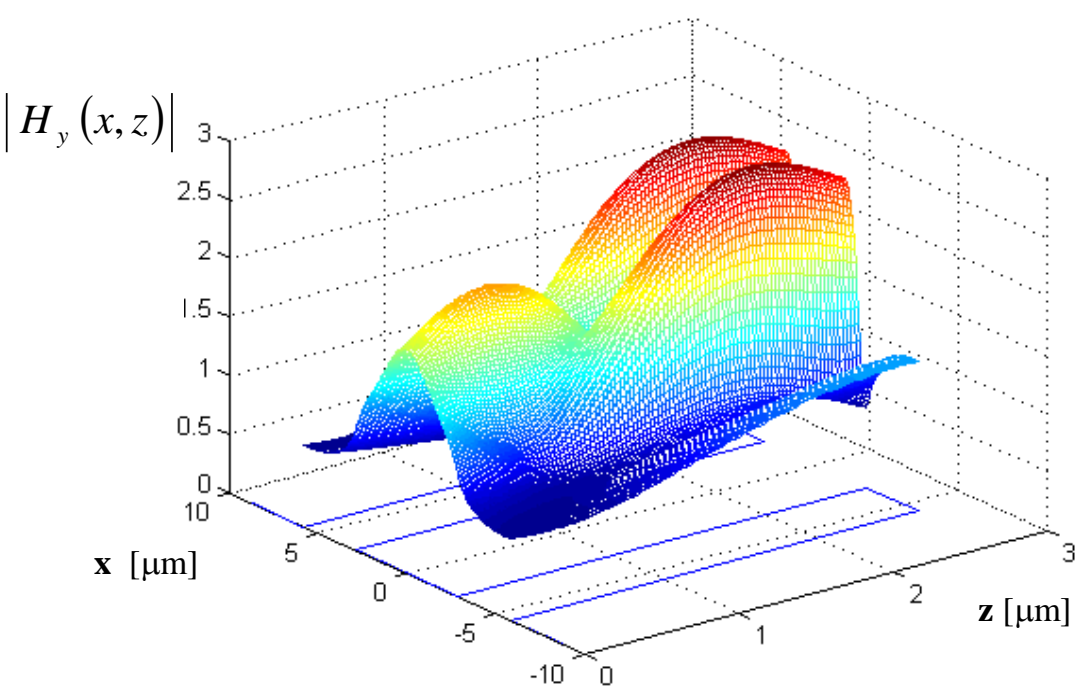

Fig. 2 Magnetic field magnitude inside the micro-lens groove. The groove profile is shown in the horizontal plane. Polarization TM 


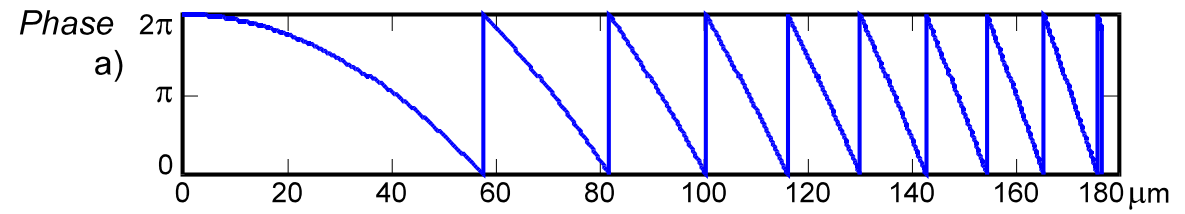

b)

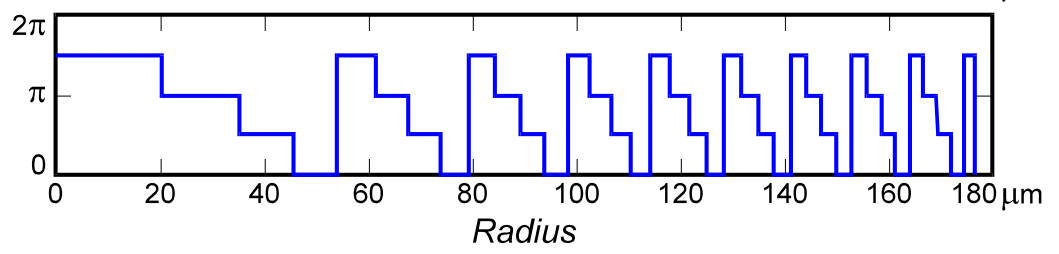

Fig. 3. Phase of the diffractive lenses: a) Fresnel lens, b) its four-phase-level approximation

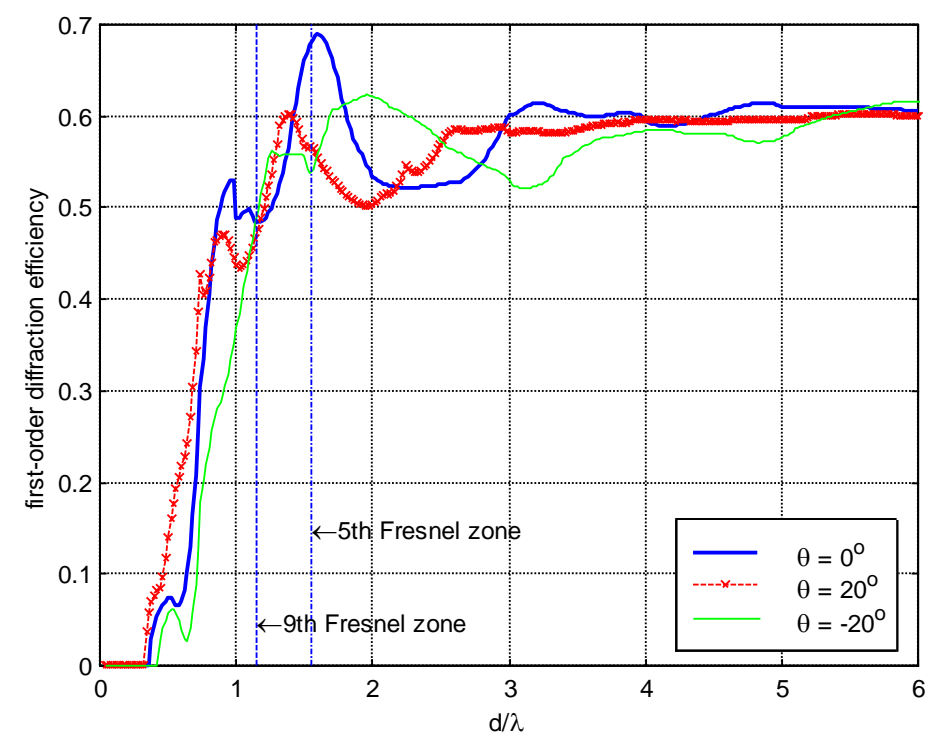

Fig. 4. Zonal efficiencies of the four-phase-level Fresnel lens averaged over both states of polarization

\section{Electromagnetic analysis of binary refractive micro-lenses}

Upper limit on the numerical aperture of the binary refractive LWIR micro-lenses is set by technological difficulties that arise in fabrication of the micro-lenses of large sag. The main challenge is an anisotropic and precisely controlled very deep (a few micrometers in each step, 10 and more micrometers total height) substrate and planarisation layer etching. The sag of the binary refractive lenses increases with the detector array pixel size for a fixed numerical aperture of the lens. The LWIR micro-lens sag is limited to teens of micrometers due to fabrication feasibility, thus it is difficult to make the binary-refractive-micro-lenses for LWIR FPA of pixel size larger than about $60 \mu \mathrm{m}$. On the other side, the pixel should be larger than the objective focal spot.

We have designed a binary approximation of aplanatic lens made of CdZnTe. Its diameter was $50 \mu \mathrm{m}$, numerical aperture - 1.8, sag -14 $\mu \mathrm{m}$. The micro-lens was illuminated through the $F / 2$ objective. Electromagnetic analysis of focusing through this micro-lens 
enabled us to study its diffraction efficiency dependence on the fabrication errors and number $\mathrm{Q}$ of relief-depth levels. The electric field distribution in the refractive micro-lens substrate is shown in Fig. 5 for $Q=8$. The Poynting vector in the focal plane normalized to the average Poynting vector of the incident wave in front of the micro-lens of $\mathrm{Q}=8$ is plotted in Fig. 6 . These results prove that eight-level binary refractive micro-lens can have numerical aperture two times higher than the binary diffractive micro-lens of the same high diffraction efficiency.

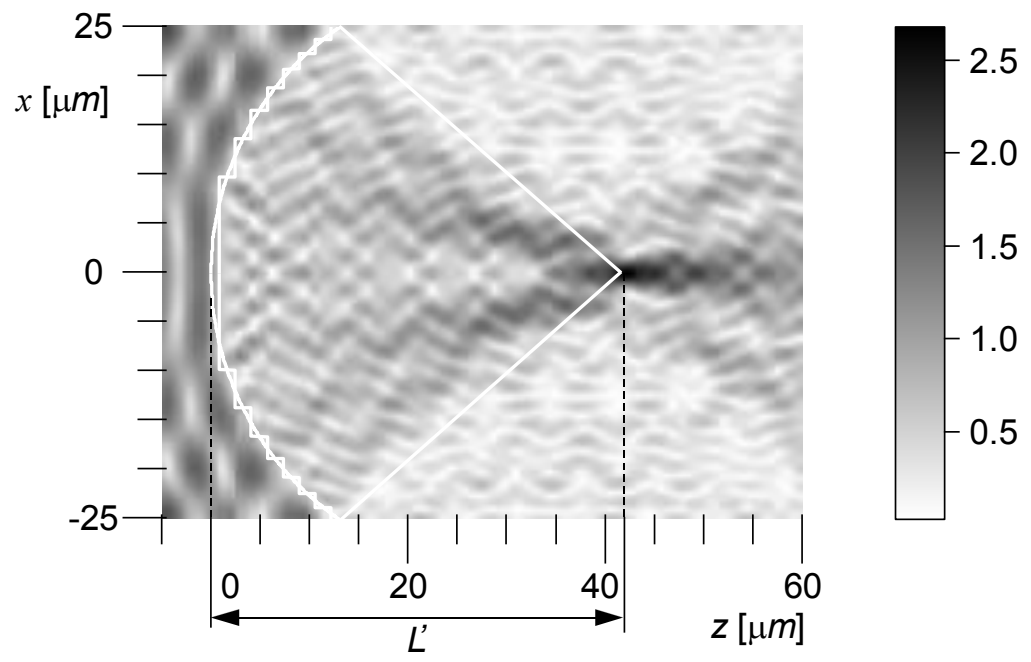

Fig. 5. Electric field magnitude in the substrate of the eight-phase-level binary refractive micro-lens

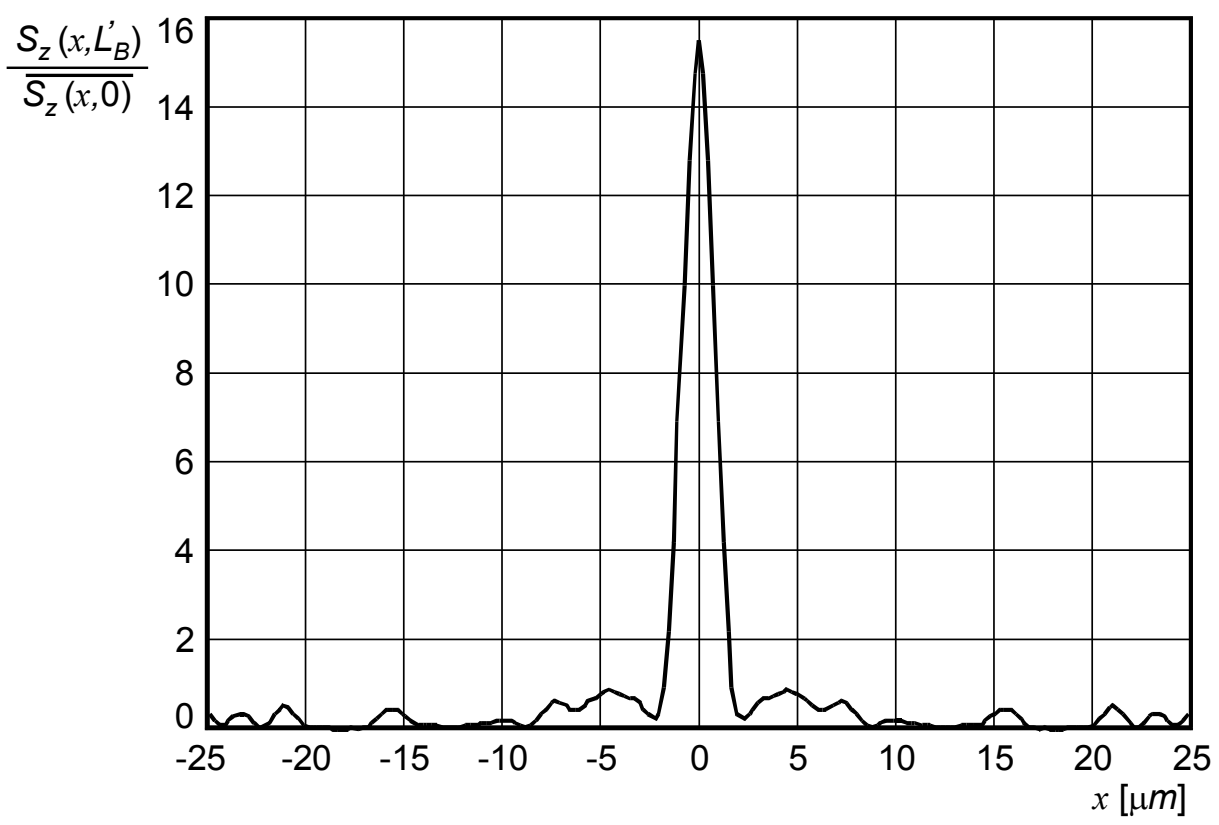

Fig. 6. Normalized Poynting vector distribution in the focal plane of eight-level binary refractive micro-lens 


\section{Conclusions}

Diffraction through the binary micro-lens array requires a formulation as an electromagnetic boundary value problem, and rigorous vector diffraction theory should be applied to solve it.

Refractive binary micro-lenses applied as a longwave infrared FPA optics can have higher numerical aperture than their diffractive counterparts of the same diffraction efficiency.

\section{ACKNOWLEGMENTS}

This paper was supported by the State Committee for Scientific Research.

\section{References}

1. D. A. Pommet, M. G. Moharam, E. B. Grann, "Limits of scalar diffraction theory for diffractive phase elements”, J. Opt. Soc. Am. A 11, pp. 1827-1834,1994.

2. Z. Sikorski, "Electromagnetic effects for the resonance-domain IR diffractive optics", Infrared Physics and Technology 41, 205-212 (2000)

3. Z. Sikorski, J. Piotrowski, and M. Grudzien,, "Electromagnetic limit of the optical gain for longwave IR diffractive optics," Optica Applicata XXX, 151-160 (2000)

4. K. Knop, "Rigorous diffraction theory for transmission phase gratings with deep rectangular grooves," J. Opt. Soc. Am. 68, pp. 1206-1210, 1978.

5. D. M. Pai and K. A. Awada, "Analysis of dielectric gratings of arbitrary profiles and thicknesses," J. Opt. Soc. Am. A8, pp. 755-762, 1991.

6. N. P. K. Cotter, T. W. Preist, and J. R. Sambles, "Scattering matrix approach to multilayer diffraction," J. Opt. Soc. Am. A 12, pp. 1097-1103, 1995. 